\title{
Structural Relaxation of Spin-Cast Glassy Polymer Thin Films as a Possible Factor in Dewetting
}

\author{
H. Richardson, C. Carelli, J.L. Keddie and M. Sferrazza
}

Department of Physics, University of Surrey, Guildford, Surrey GU2 7XH, UK

\begin{abstract}
Reiter [1] has recently reported a situation in which the dewetting of quasi-solid films is linked plastic deformation - rather than viscous flow - resulting from capillary forces. Herein we propose that, in thin films of some glassy polymers - especially poly(methyl methacrylate) (PMMA) - prepared by spin-casting from solvent, structural relaxation might impart sufficient stress to cause plastic deformation. We find that PMMA films decrease in thickness by several percent, which is sufficient to create significant stress in those cases in which the film is attached to a rigid substrate. The floating technique, which can take tens of minutes, might allow most of the structural relaxation to occur prior to dewetting experiments.
\end{abstract}

PACS. 65.40.De Thermal expansion; thermomechanical effects - 82.60.Lf Thermodynamics of solutions - 61.41.+e Polymers, elastomers and plastics

Running title: Relaxation in Glassy, Spin-Cast Polymer Thin Films

\section{Experimental Studies of Polymer Dewetting}

The wetting and dewetting of surfaces by thin liquid films are well-known phenomena that have been studied extensively [2]. Polymer thin films represent ideal systems to study such physical phenomena due to their low vapour pressure and high viscosity, which allows time-resolved experiments to be undertaken. The dewetting behaviour of thin polymer films has also attracted substantial technological interest due to the 
widespread use of polymers in coatings and packaging in which film integrity is essential and because of its potential application in patterning [3]. Recently, much attention has been paid to the dewetting behaviour of poly(styrene) (PS) films on silicon or on another polymer [4-12], such as rubbery poly(dimethyl siloxane) (PDMS) [1,11,12]. In other studies, the spinodal dewetting of thin poly(methyl methacrylate) (PMMA) films deposited on a PS substrate has been extensively investigated [13,14,3].

In most of these studies, the thin polymer films are produced by the spin-cast technique: a low concentration polymer solution in toluene (or similar solvent) is spin-cast on top of either a silicon substrate or a PDMS brush directly [1,10-12], or else spin-cast on a glass or a mica foil, floated on the surface of water and then deposited onto a silicon or a polymer substrate $[8,9,13,14,3]$. The dewetting process, whether spinodal or by nucleation, is then probed by various techniques shortly after the film deposition by annealing at temperatures near and above the glass transition temperature, $T_{g}$. In most instances, the spin-cast film is not annealed prior to the dewetting experiment to allow the glass to reach an equilibrium structure and to evaporate any remaining solvent. For example, in dewetting studies of the PMMA/PS bilayer system [13,14,3], a PMMA thin film is deposited by the "floating" technique onto the PS substrate without prior annealing. The bilayer system, PMMA/PS/Si, is then annealed for a few hours in vacuum at a temperature well below the $T_{g}$. In only a few cases has the spin-cast film been annealed to allow structural relaxation and solvent loss prior to the dewetting experiment, such as in experiments where PMMA films were annealed at $120{ }^{\circ} \mathrm{C}$ for 24 hours [15]. We note that the initial state of the films in most of these dewetting experiments is inherently metastable [16]. In this paper, we point out that the spin-cast films are likely to undergo structural relaxation while simultaneously dewetting their substrate.

\section{Structural Relaxation of Spin-Cast Glassy Thin Films}

For a given temperature, a glass has a characteristic structural relaxation time $\tau$. As a quenched glass is re-heated, $\tau$ decreases exponentially with temperature. At some point, 
$\tau$ will be comparable to the timescale of the experimental observation, $\tau_{\text {exp }}$, and volume relaxation will be observable. This phenomenon, particularly as it relates to oxide glasses, has been thoroughly studied [17]. During a quench (or spin-casting), $\tau_{\exp }$ is very short, and a large volume is "frozen in" because there is not sufficient time for the relaxation. A sub- $T_{\mathrm{g}}$ anneal, however, will lower $\tau$, making it comparable to the $\tau_{\exp }$ of the technique used to probe the relaxation (e.g. ellipsometry or calorimetry). The volume will then fall until it reaches the value it would have otherwise obtained by slow cooling from the melt (i.e. corresponding to a longer $\tau_{\text {exp }}$ ), often referred to as an "equilibrium" volume. In summary, when a glass is quenched from the melt, its resulting volume is greater than what it would have, if it had been cooled slowly. Upon re-heating, the glass will undergo structural relaxation and a volume decrease to reach its equilibrium volume $[17,18]$.

When a polymer is spin-cast at room temperature, solvent is rapidly evaporated, and as a result of the rising glass transition temperature $T_{g}$, the solution can pass from the rubbery state to the glassy state. In this way, there is a direct analogy between quenching from the melt and the spin-casting process [19-21]. Spin-cast, glassy polymer films might therefore be expected to show a volume decrease resulting from structural relaxation when they are heated near their $T_{g}$. (Kawana and Jones [22] have described the opposite effect in which slowly-cooled films are re-heated quickly and expand to reach their equilibrium volume. This phenomenon occurs at a temperature near the $T_{\mathrm{g}}$ at which $\tau$ is comparable to $t_{\text {exp. }}$ )

In addition to exhibiting slow structural relaxation, spin-cast films are suspected of retaining solvent for unexpectedly long times. As pointed out by Reiter and de Gennes [16], the compression of a glass creates an energy barrier to solvent loss in those cases in which voids are not created. (Void creation increases the surface area and so is not thermodynamically favorable.) We have found experimentally that there is a marked slowing down of solvent loss from a spin-cast PMMA thin film when its $T_{g}$ rises above the temperature of the ambient [21]. Thickness changes associated with solvent loss occurred over several hours at room temperature. 
In order to gain a quantitative insight into thin film stability, experiments were conducted to measure the amount of solvent that remains in glassy polymer thin films after spincasting. We measured the mass of a freshly spin-cast PMMA on a gold surface using a quartz-crystal microbalance (QCM) (RQCM, Maxtek, Inc., USA) while simultaneously measuring the film thickness using spectroscopic ellipsometry (VASE, J.A. Woollam, USA $)$ at room temperature $\left(25^{\circ} \mathrm{C}\right)$. Films of PMMA $\left(M_{\mathrm{w}}=1.42 \times 10^{5} \mathrm{~g} \mathrm{~mol}^{-1}\right.$ and $\left.M_{\mathrm{w}} / M_{\mathrm{n}}=1.01\right)$ were spin-cast directly onto the gold surface from a solution in toluene. The combined use of the two techniques is able to determine whether volume change always accompanies solvent evaporation or if there is mass loss without film shrinkage, providing evidence for void formation.

In the QCM data analysis, the well-known Sauerbrey equation [23] is used to relate the frequency shift, $\Delta f$, of the resonant frequency of the quartz crystal to the change in a film's mass per unit area, $\Delta m$ :

$$
\Delta m=-\frac{Z_{q} \Delta f}{2 f_{o}{ }^{2}},
$$

where $f_{o}$ is the resonant frequency of a bare quartz surface and $Z_{q}$ is a constant equal to the product of the density of quartz and the speed of sound in quartz $\left(Z_{q}=8.8 \times 10^{6} \mathrm{~kg} \mathrm{~m}^{-}\right.$ $\left.{ }^{2} \mathrm{~s}^{-1}\right)$. Hence, an increase in $\Delta f$ corresponds to a loss of mass, such as would accompany solvent evaporation. In the ellipsometry experiments, full spectroscopic scans at a fixed angle-of-incidence were made at regular time intervals. Accurate values of film thickness were thereby obtained.

The results from a typical experiment are plotted in Figure 1. For the first $200 \mathrm{~min}$. after spin-casting, there is a linear relationship between mass loss and film shrinkage, which is consistent with relaxation of the glass as solvent is evaporated, implying that excess volume or nanovoids are not being created. There is a small departure from this monotonic relationship at later times, which is deserving of further study. After 1480 min. ( $\approx 24 \mathrm{hr}$.), there is a stabilisation of the mass but further film shrinkage of more than $5 \mathrm{~nm}$. At this later time, there is thus evidence that the structure of the glass is relaxing to 
eliminate excess volume. Solvent evaporation proceeds more slowly than what is expected when considering the diffusion coefficient of toluene in glassy PMMA [24]. Based on the amount of the observed film shrinkage, we estimate that spin-cast film initially contained 6 vol.\% toluene. This concentration of solvent is predicted [25] to depress the glass transition temperature of the film by $31 \mathrm{~K}$ to a value of $352 \mathrm{~K}$. Hence, the film would be rubbery when annealed at values far below the $T_{g}$ of the neat polymer.

There is another important ramification of this result for studies of dewetting. If solvent is lost from the PMMA film in a PMMA/PS bilayer, there is a strong possibility that some of this solvent will be absorbed into the PS film. The solvent would then act as a plasticiser in the PS, with the potential of creating a rubbery interface, rather than a glassy one.

Our experiments have found that the structure of poly(styrene) spin-cast thin films is much more stable and does not undergo more than about $1 \%$ thickness change (e.g. a decrease of $1.7 \mathrm{~nm}$ in a $148 \mathrm{~nm}$ film) even when annealed above $T_{g}$ and cooled to room temperature again. This finding is consistent with the results of other workers $[3,26,27,28]$ who have all seen typically a decrease of only 2 or $3 \AA$ in films as thick as $30 \mathrm{~nm}$. Since both PMMA and PS have comparable glass transition temperatures and bulk moduli, this result suggests that there are some other important factors in determining the structural relaxation after quenching. In studies in the literature that consider the dewetting of PS, the possible effects of structural relaxation are not apparently very relevant. (Incidentally, the literature often refers to negative expansivity $[27,28]$ in thin films. Such decreases in film thickness resulting from structural relaxation are seen at a constant temperature also.)

\section{Stress Generation during the Shrinkage of Pinned Films}

The amount of volume relaxation in the depth of the film (i.e. the "vertical" direction) should be correlated with the relaxation in the plane of the film. If the film was pinned to the substrate, a greater thickness decrease would be required to achieve a certain volume decrease, compared to the case in which the film is able to shrink laterally. Hydrogen bonding between surface hydroxyl or silanol groups and the PMMA's methacrylate group [29] might be sufficient to pin the PMMA film to the substrate and thus prevent lateral 
slippage. In most dewetting experiments reported in the literature, however, a PMMA film is deposited onto a PS film. On the non-polar surface of PS, in comparison to the oxide, the PMMA might be able to slip and therefore relax in the plane of the film, causing a smaller change in thickness.

The problem of stress generation in a film that is prevented from shrinking laterally is analogous to the problem of a thermal expansion mismatch between a film and substrate [30]. For a strain $\varepsilon$ resulting from shrinkage in the film, the mean stress $\sigma$ to oppose the shrinkage is given as $\sigma=\frac{E \varepsilon}{(1-v)}$

where the Poisson ratio $v$ of PMMA is about 0.35 [31], and its elastic modulus $E$ is typically $3 \mathrm{GPa}$ [32]. Hence, for $\varepsilon=0.08$, which is the maximum expected in spin-cast PMMA, $\sigma$ is about $370 \mathrm{MPa}$. This level of stress - if it is not relieved - is sufficient to cause plastic deformation in PMMA. It is therefore worthwhile to consider the likelihood of such stress developing in systems used to study dewetting.

This idea of plastic deformation creating dewetting patterns is not new. Reiter [1] has commented recently that the dewetting that is observed in quasi-solid PS films near their $T_{g}$ is not consistent with a viscous flow mechanism. Instead, he proposed that capillary forces are sufficient to cause plastic deformation. In bilayers of PMMA on PS, the stress resulting from the structural relaxation of a PMMA film could be sufficient to likewise induce plastic deformation during the dewetting process.

\section{Influence of the Floating Procedure on Film Shrinkage in PMMA} Films

The important question is whether shrinkage stresses are present in films used in dewetting experiments. As stated in Section 1, PMMA films are often deposited onto 
substrates after floating on the surface of water. This deposition procedure typically takes tens of minutes, during which time structural relaxation is expected to occur. It might also be possible for the water to be absorbed into the PMMA and then lower its $T_{g}$. (It has been found elsewhere [33] that PMMA absorbs more than 2 vol.\% water at high relative humidities.) Finally, it is not known if the PMMA will slip laterally across the surface of a glassy polymer substrate. To explore these ideas further, preliminary experiments were conducted.

We have measured the decreasing thickness in spin-cast PMMA thin films during annealing at $55{ }^{\circ} \mathrm{C}$. This temperature was chosen because it induces relaxation that occurs over a timescale of several hours, making it ideal for experiments. The relaxation of a spin-coated PMMA film, nominally $70 \mathrm{~nm}$ thick, was probed after preparation by one of three ways: spin-casting directly onto a silicon substrate; depositing from the surface of water onto a silicon substrate after floating off of glass; and depositing from the surface of water onto a $100 \mathrm{~nm}$, annealed PS film after floating off of glass. The results are presented in Figure 2. The thickness of the film is normalised by the value after 1000 min. of annealing for ease of comparison. For all three experiments, the time of spin-casting was defined as the zero point. The time axis has been shifted for the films that were floated onto the surface of water to take into account the time spent in the deposition procedure. The thickness of the film that was cast directly onto silicon decreases from an initial value of $72.3 \mathrm{~nm}$ to a value of $69.6 \mathrm{~nm}$ at $1000 \mathrm{~min}$. In contrast, the fractional decrease in thickness observed in the films that had been "floated" onto their substrate is much smaller overall but agrees well during the time period of the experiment.

These results show that the shrinkage on a silicon substrate is comparable to what is found on both PS and silicon substrates after the floating procedure; the data overlay very well. The shrinkage during the floating procedure itself cannot be measured, but by comparing to the shrinkage of the spin-cast film, we suspect that substantial shrinkage took place prior to annealing the film at $55{ }^{\circ} \mathrm{C}$. It is important to remember that the floating procedure takes place at room temperature. As relaxation is expected to be 
slower at room temperature, there is the possibility that the relaxation in the films during the floating procedure is faster than when on a silicon substrate. Plasticisation by water might be one cause of any enhanced relaxation rate. A liquid substrate, in comparison to a rigid solid, might also enable faster relaxation.

To summarise, we suggest that structural relaxation in spin-cast thin films is capable of generating stress in those cases in which the film is pinned to a substrate. This stress has the potential to cause plastic deformation and perhaps hole formation. The volume changes in spin-cast, unannealed PMMA films are greater than what are found in PS films. The positive outcome of our experiments in relation to dewetting studies is that there probably is not a high level of stress generated in PMMA/PS bilayers, because structural relaxation occurs during the floating procedure. However, structural relaxation is still relevant to consider in experiments in which spin-cast glassy films are deposited directly onto a substrate.

Ackowledgements: We gratefully acknowledge funding from the UK's Engineering and Physical Sciences Research Council for the support of H. Richardson and C. Carelli and for the purchase of the ellipsometer. 
Published in Eur Phys J E Soft Matter (2003) 12(3):437-440

\section{References}

[1] G. Reiter, Phy. Rev. Lett. 87,186101 (2001).

[2] P. G. de Gennes, Reviews of Modern Physics 57, 827, (1985).

[3] A. Higgins and RAL Jones, Nature 404, 476 (2000)

[4] G. Reiter, Phy. Rev. Lett. 68, 75, (1992)

[5] P. Lambooy, K. C. Phelan, O. Haugg and G. Krausch, Phy. Rev. Lett. 76, 1110,(1996).

[6] S. Qu, C. J. Clarke, Y. Liu, M. H. Rafailovich, J. Sokolov, K. C. Phelan and G. Krausch, Macromolecules 30, 3640, (1997).

[7] G. Krausch, J. of Phy. -Cond. Mat. 9, 7741, (1997)

[8] R. Seemann, S. Herminghaus and K. Jacobs, J. of Phy.-Cond. Mat. 13, 4925, (2001)

[9] R. Seemann, S. Herminghaus and K. Jacobs, Phy. Rev. Lett. 86, 5534, (2001)

[10] R. Xie, A. Karim, J. F. Douglas, C. C. Han and R. A. Weiss, Phy. Rev. Lett. 81, 1251, (1998)

[11] G. Reiter, R. Khanna and A. Sharma, Phy. Rev. Lett. 85, 1432, (2000)

[12] G. Reiter, M. Sferrazza, P. Damman, Eur. Phys. J. E, in press

[13] M. Sferrazza, M. Heppenstallbutler, R. Cubitt, D. G. Bucknall, J. Webster and R. A. L. Jones, Phy. Rev. Lett. 81, 5173, (1998)

[14] A. Higgins M. Sferrazza, RAL Jones, PC Jukes, JS Sharp, LE Dryden and J. Webster, Eur. Phys. J. E. 8, 137 (2002)

[15] H. Wang, RJ Composto, J. Chem. Phys. 113, 10386 (2000)

[16] G. Reiter and P de Gennes, Eur. Phys. J E 6, 25 (2001)

[17] I.M. Hodge, J. Non-Cryst. Sol. 169, 211 (1994).

[18] J.M. Hutchinson, Prog. Polym. Sci. 20, 703 (1995).

[19] S.G. Croll, J. Appl. Pol. Sci. 23, 847 (1979).

[20] G.B. McKenna, J. Phys. IV France, 10, 53 (2000).

[21] H. Richardson, M. Sferrazza, JL Keddie, Eur. Phys. J E Direct, in press

[22] S.Kawana and R.A.L. Jones, Eur. Phys. J. E 10, 223 (2003).

[23] C. Lu, in Applications of Piezoelectric Quartz Crystal Microbalances, edited by C. Lu and A.W. Czanderna (Elsevier, Amsterdam, 1984).

[24] B.-G. Wang, T. Yamaguchi, S.-I. Nakao, J. Polym. Sci. B: Polym. Phys. 38, 846 (2000).

[25] F.N. Kelley and F. Bueche, J. Polym. Sci. 50, 549, (1961).

[26] G. Reiter, Macromolecules 27, 3046 (1994).

[27] M.Mukherjee, M. Bhattacharya, and M.K. Sanyal, Phys. Rev. E 66, 061801 (2002).

[28] T. Kanaya, T. Miyazaki, J. Watanabe, K. Nishida, H. Yamano, S. Tasaki, D.B.

Bucknall, Polymer 44, 3769 (2003).

[29] J.L. Keddie, R.A.L. Jones, R.A. Cory, Faraday Disc. 98, 219 (1994). 
[30] S. Timoshenko and S. Woinowsky-Krieger, Theory of Plates and Shells, 2nd edn. (McGraw-Hill, New York, 1959).

[31] A. Domack and D. Johannsmann, J. Appl. Phys., 83, 1286 (1998).

[32] A.-C. Saby-Dubreuil, B. Guerrier, C. Allain, and D. Johannsmann, Polymer, 42, 1383 (2001).

[33] W.-L. Chen, K.R. Shull, T. Papatheodorou, D.A. Styrkas and J.L. Keddie, Macromolecules 32, 136 (1999).

Figure Captions

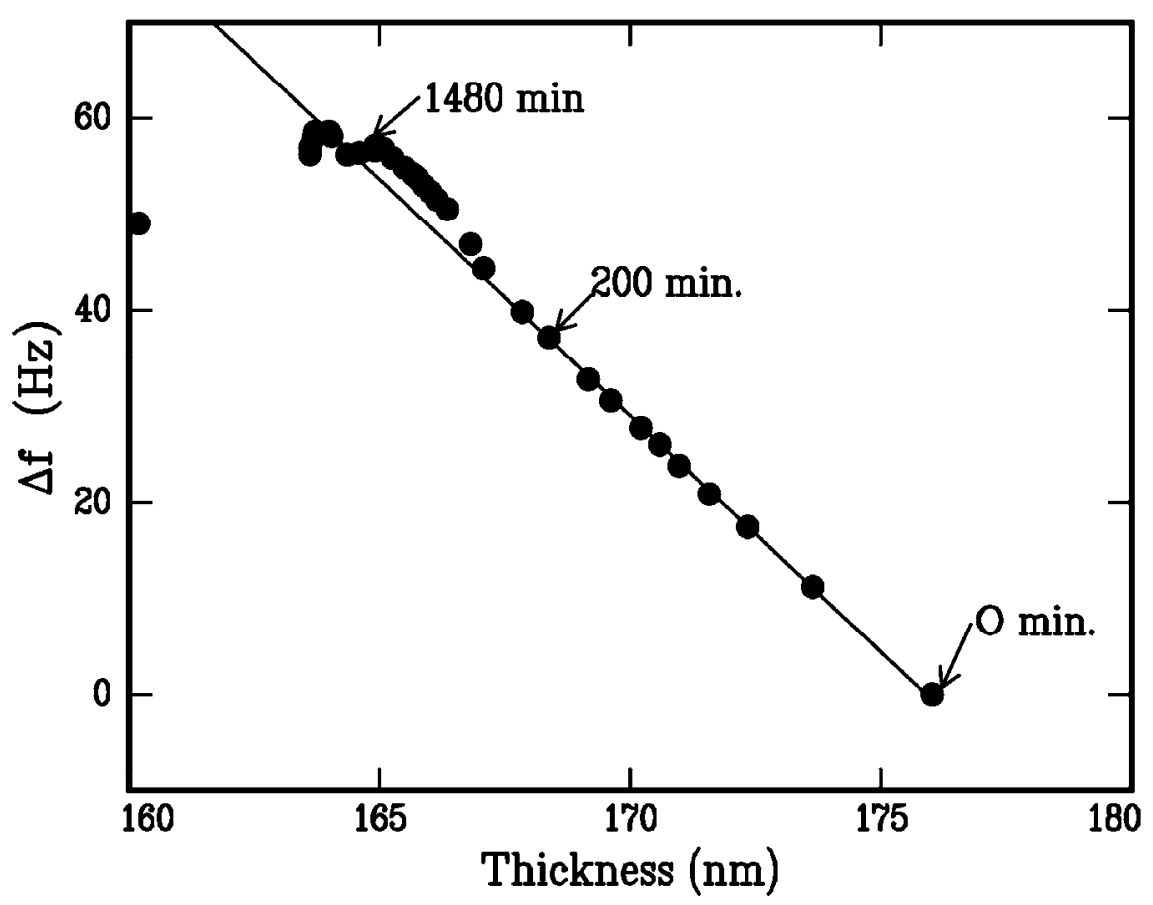

Fig. 1. Correlation of mass loss (indicated by the increasing frequency of the quartz crystal) and thickness change in a spin-cast PMMA film at a temperature of $25^{\circ} \mathrm{C}$. 


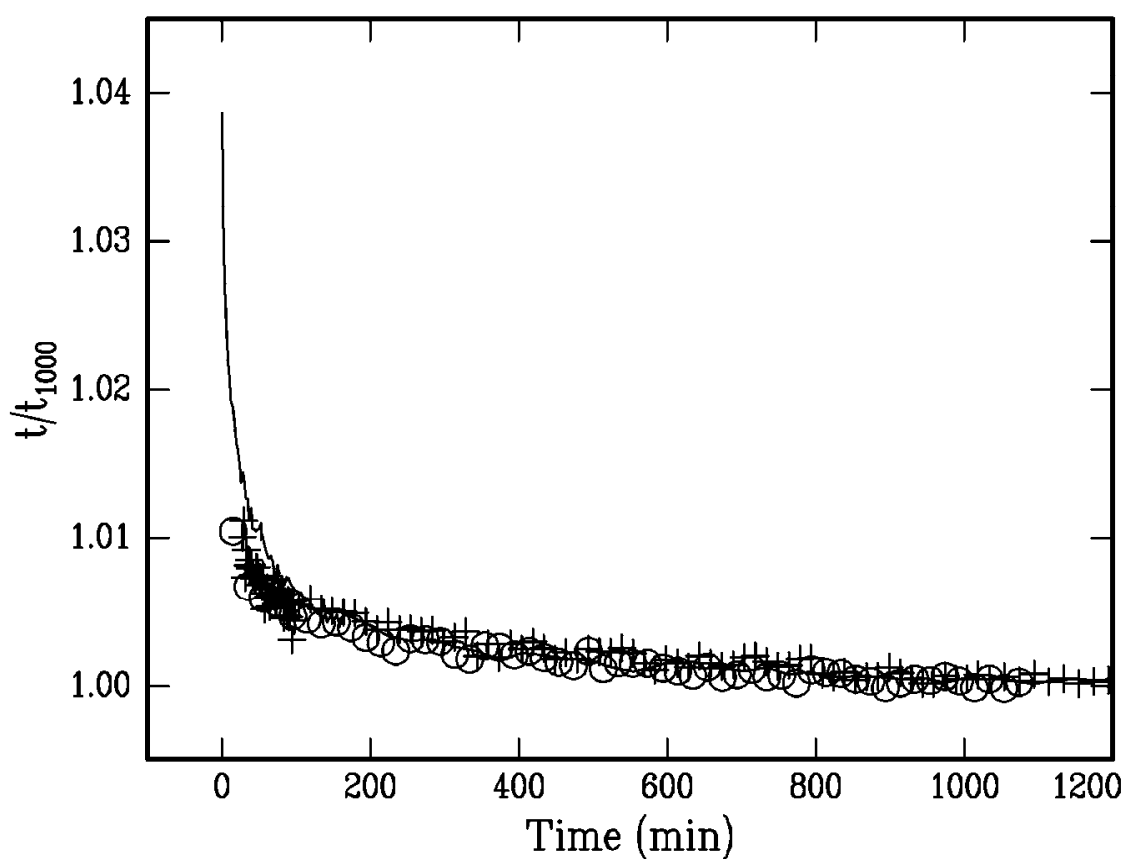

Fig. 2. Structural relaxation of PMMA at a constant temperature of $55^{\circ} \mathrm{C}$ in thin films spin-cast from toluene onto silicon ( - ), floated onto a $100 \mathrm{~nm}$ PS film on silicon (+), and floated onto silicon (O). All films are nominally $70 \mathrm{~nm}$ thick. Thickness values are divided by the thickness at $1000 \mathrm{~min}$. Inset: The same data are shifted vertically for clarity. Filled circles $\left({ }^{\bullet}\right)$ show the data from PMMA spin-cast directly onto silicon. 